\title{
Optical turbulence and transverse rogue waves in a cavity with triple-quantum-dot molecules
}

\author{
M. Eslami, ${ }^{1,}{ }^{*}$ M. Khanmohammadi, ${ }^{2}$ R. Kheradmand, ${ }^{2}$ and G.-L. Oppo ${ }^{3}$ \\ ${ }^{1}$ Young Researchers and Elite Club, Tabriz Branch, Islamic Azad University, Tabriz, Iran \\ ${ }^{2}$ Photonics Group, RIAPA, University of Tabriz, Tabriz, Iran \\ ${ }^{3}$ Department of Physics, University of Strathclyde, Glasgow G4 ONG, Scotland, EU
}

(Received 9 April 2017; published 20 September 2017)

\begin{abstract}
We show that optical turbulence extreme events can exist in the transverse dynamics of a cavity containing molecules of triple quantum dots under conditions close to tunneling-induced transparency. These nanostructures, when coupled via tunneling, form a four-level configuration with tunable energy-level separations. We show that such a system exhibits multistability and bistability of Turing structures in instability domains with different critical wave vectors. By numerical simulation of the mean-field equation that describes the transverse dynamics of the system, we show that the simultaneous presence of two transverse solutions with opposite nonlinearities gives rise to a series of turbulent structures with the capability of generating two-dimensional rogue waves.
\end{abstract}

DOI: 10.1103/PhysRevA.96.033836

\section{INTRODUCTION}

Although they are called by different names, rogue, freak, killer, and extreme waves and even rogons and freakons, rogue waves (RWs) are characterized by amplitudes that are much higher compared to the long-time average height of the oscillations in a medium [1]. These waves, occurring unexpectedly, are typically accompanied by wide and/or deep troughs before or after the event. Such occurrences were first studied in oceanography, where RWs have been reported to damage or even destroy large vessels without warnings. Then the concept of RWs gradually moved into other fields, like optics, matter waves, and superfluidity, in the last decade [1,2].

As in any other newly introduced phenomenon in natural sciences, there is still a lack of uniformity in the definition and characterization of RWs. While oceanographers use the basic criterion of their being 2-2.5 times larger than the average height of other waves, several definitions of RWs have been used in different contexts and physical systems [3-6]. A common and very useful method for studying the occurrences of RWs is the probability density function (PDF), which determines the shape of the intensity (or amplitude) distribution of events in a long enough time series. Normally the distribution is of a Gaussian shape unless extreme events occur, with the result of elongating its tail. Predictability of RWs is also a recently debated feature with obvious applications in the control or forecasting of rare events. Although it is widely accepted that RWs are chaotic to a large extent, there have been some studies that successfully argue their deterministic character in optical systems [4] and even suggest ways to foretell their occurrence $[5,7,8]$. The key element for the characterization of extreme events is the understanding of the underlying mechanism leading to the formation of optical RWs [6]. In nonlinear physics, a variety of mechanisms have been identified $[1,6]$ including, more recently, breather and soliton turbulence in conservative systems [9].

In the context of transverse optics in resonators, the two building blocks of optical turbulence and extreme events are nonlinearity and modulation instability, which are typically

\footnotetext{
*m.eslami@ tabrizu.ac.ir
}

present in Kerr cavities and semiconductor systems in the presence of diffraction and/or dispersion. Different mechanisms for RWs in these systems have been identified: vortex-mediated turbulence [10,11], spatiotemporal chaos [12], and even cavity solitons [13]. On the other hand, the complexity and multistability of solutions are generic properties of atomic systems with enhanced material coherence provided by phenomena like electromagnetically induced transparency and coherent population trapping $[14,15]$. These systems display competing nonlinearities and lead to the formation of extended and localized optical structures when additional coupling lasers induce modifications to the nonlinear properties typical of atomic systems.

Important features of atomic systems, such as sharp and well-defined energy levels, are also provided effectively by semiconductor quantum dots (QDs) whose electronic degrees of freedom are discretized due to the confinement of electrons and holes, leading to promising nanostructures. Furthermore, quantum dot molecules (QDMs) can be formed when QDs couple to each other by a tunneling mechanism which is controllable by an external electric field (gate voltage), eliminating the need for any additional coupling laser beam [16]. Modification of the quantum states is then possible by the novel interaction avenue displayed by coupled QDMs under the settings of tunneling-induced transparency. In this paper we focus on a triple QDM in a cavity to show that tunneling-induced transparency is a suitable element to form transverse spatial structures and competition of nonlinearities. We extend the complexity to optical turbulent regimes and RWs. In Sec. II we present the model equations and discuss steady states. Then we turn our attention, in Sec. III, to spatial structures in two different but overlapping instability domains. Section IV explains the nature of competing nonlinearities, followed by discussions of turbulent structures and RWs. We present evidence for a previously unreported mechanism for the generation of RWs in nonlinear optics: spatial competition of periodic patterns. Conclusions are outlined and final remarks made in Sec. V.

\section{THE MODEL}

The system of interest here is a Fabry-Perot cavity filled with triple-quantum-dot molecules, where the dots in 

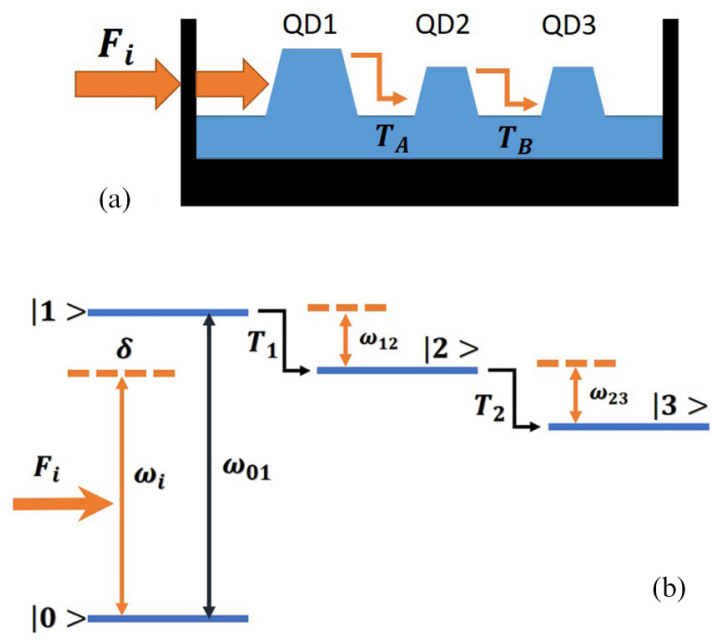

FIG. 1. (a) Cavity configuration for a typical triple-quantum-dot molecule with an injected pump and tunnelings. (b) Energy-level structure for dots in the molecule.

individual molecules are coupled by electric gates as shown in Fig. 1. Unlike atomic systems, where the presence of coupling laser fields is necessary for the establishment of electromagnetically induced transparency and coherent population trapping, semiconductor quantum dots can use voltage gates to control the tunneling condition of electrons between energy states, which can be tuned to achieve tunneling-induced transparency. Such a medium in a cavity can be described by a single mean-field equation,

$$
\partial_{t} F=F_{i}-[(1+i \theta)+i \Sigma \chi(F)] F+i \nabla^{2} F,
$$

where $F$ is the slowly varying amplitude of the electric field, $F_{i}$ is the injected pump field, with a frequency $\omega_{i}$ close to that of the transition $|0\rangle \rightarrow|1\rangle$, and the detuning term $\theta$ is the frequency difference between the cavity resonance and $\omega_{i}$. The diffraction term is given by the Laplacian operator in two transverse dimensions and time is normalized to the photon lifetime in the cavity.

$\Sigma$ is the cooperative parameter directly proportional to the number density of quantum dot molecules $N$ through

$$
\Sigma=\frac{N \mu_{01}^{2} k L}{\hbar \Gamma \epsilon_{0} T},
$$

where $\mu_{01}$ is the transition dipole moment, $k$ the wave number of the cavity field, $L$ the length of the cavity, $\Gamma$ the line width, $\epsilon_{0}$ the permittivity of free space, and $T$ the cavity mirror transmittivity. $\chi(F)$ is the complex susceptibility, which contains all the medium-related details due to the off-diagonal density matrix elements when multiplied by the field amplitude $F$. For simplicity we consider a noninteracting situation among individual QDMs inside the cavity.

For the configuration shown in Fig. 1(b), the Hamiltonian of the system can be written as

$$
H=H_{0}+H_{I}+H_{T}
$$

where the unperturbed, interaction and tunneling Hamiltonian terms are, respectively,

$$
\begin{gathered}
H_{0}=\hbar \sum_{j=0}^{3} \omega_{j}|j\rangle\langle j|, \\
H_{I}=\frac{1}{2} F_{i} \mu_{01}|0\rangle\langle 1| e^{\left(i k . x-i \omega_{i} t\right)}+\text { H.c. } \\
H_{T}=T_{A}|2\rangle\left\langle 1\left|+T_{B}\right| 3\right\rangle\langle 2|+\text { H.c., }
\end{gathered}
$$

where H.c. stands for Hermitian conjugate. In the Hamiltonian terms above, $j$ represents the respective energy level, $x$ is the longitudinal coordinate, and $T_{A}$ and $T_{B}$ denote the electron tunneling matrix elements for the $|1\rangle \rightarrow|2\rangle$ and $|2\rangle \rightarrow|3\rangle$ transitions, respectively. Hole tunneling is neglected in the considered time scales.

After some exhaustive but straightforward steps, the following set of relations from density matrix equations of motion can be obtained under the rotating-wave approximation and steady-state condition:

$$
\begin{aligned}
-i F & =\left(i \delta-\gamma_{01}\right) \rho_{01}+i T_{A} \rho_{02}, \\
0 & =\left\{i\left(\delta-\omega_{12}\right)-\gamma_{20}\right\} \rho_{02}-i F \rho_{12}+i T_{A} \rho_{01}+i T_{B} \rho_{03}, \\
0 & =\left\{i\left(\delta-\omega_{12}-\omega_{23}\right)-\gamma_{30}\right\} \rho_{03}-i F \rho_{13}+i T_{B} \rho_{02}, \\
0 & =-\left(i \omega_{12}+\gamma_{12}\right) \rho_{12}-i F^{*} \rho_{02}+i T_{B} \rho_{13}, \\
0 & =-\left(i \omega_{23}+\gamma_{23}\right) \rho_{23}-i T_{A} \rho_{13}, \\
0 & =-\left(i \omega_{13}+\gamma_{13}\right) \rho_{13}-i F^{*} \rho_{03}-i T_{A} \rho_{23}+i T_{B} \rho_{12},
\end{aligned}
$$

where $\delta$ is the frequency mismatch between the injected field and the $|0\rangle \rightarrow|1\rangle$ transition, here considered to be positive for a self-focusing nonlinearity. $\omega_{12}$ is the frequency difference between level $|1\rangle$ and level $|2\rangle$ and $\omega_{23}$ is that between $|2\rangle$ and $|3\rangle$. Both of the level separations are managed by electric gates which give control over the occupation of levels $|2\rangle$ and $|3\rangle$ [17]. We have also assumed the value of 1 for the relaxation rate $\gamma_{01}$ and $10^{-3} \gamma_{01}$ for all others. Once these coupled equations are solved for $\rho_{01}$ and substituted in Eq. (1) according to $\chi(F)=\rho_{01} / F$, the nonlinear part of the mean-field equation is obtained. We note that both $\rho_{01}$ and the susceptibility $\chi(F)$ are complex functions of the intracavity field $F$. Dispersion and absorption behaviors of the derived nonlinearity are shown in Fig. 2(a), where vanishing absorption is identified at $\delta=0.37$. Details of such calculations for a variety of QDMs can be found in [17-19].

Investigations of the homogeneous steady states $\left(\partial_{t}=0\right.$ and $\nabla^{2}=0$ ) show a departure from bistable to multistable behavior for the input-output relation when the population in level $|2\rangle$ increases, as shown in Fig. 2(b). Therefore, we use the parameter $\omega_{12}$ (or, equivalently, the gate voltage) as the control parameter in the dynamical simulations. Another experimental possibility of choosing a control parameter would be the frequency mismatch between the injected field and the $|0\rangle \rightarrow|1\rangle$ transition, $\delta$, while $\omega_{12}$ is kept fixed. However, as detailed in [17], a better electron transfer between the levels is obtained when the levels are off-resonance, thus making the idea of a fixed $\omega_{12}$ with a varying input pulse frequency less effective for observing clear results in practice. 

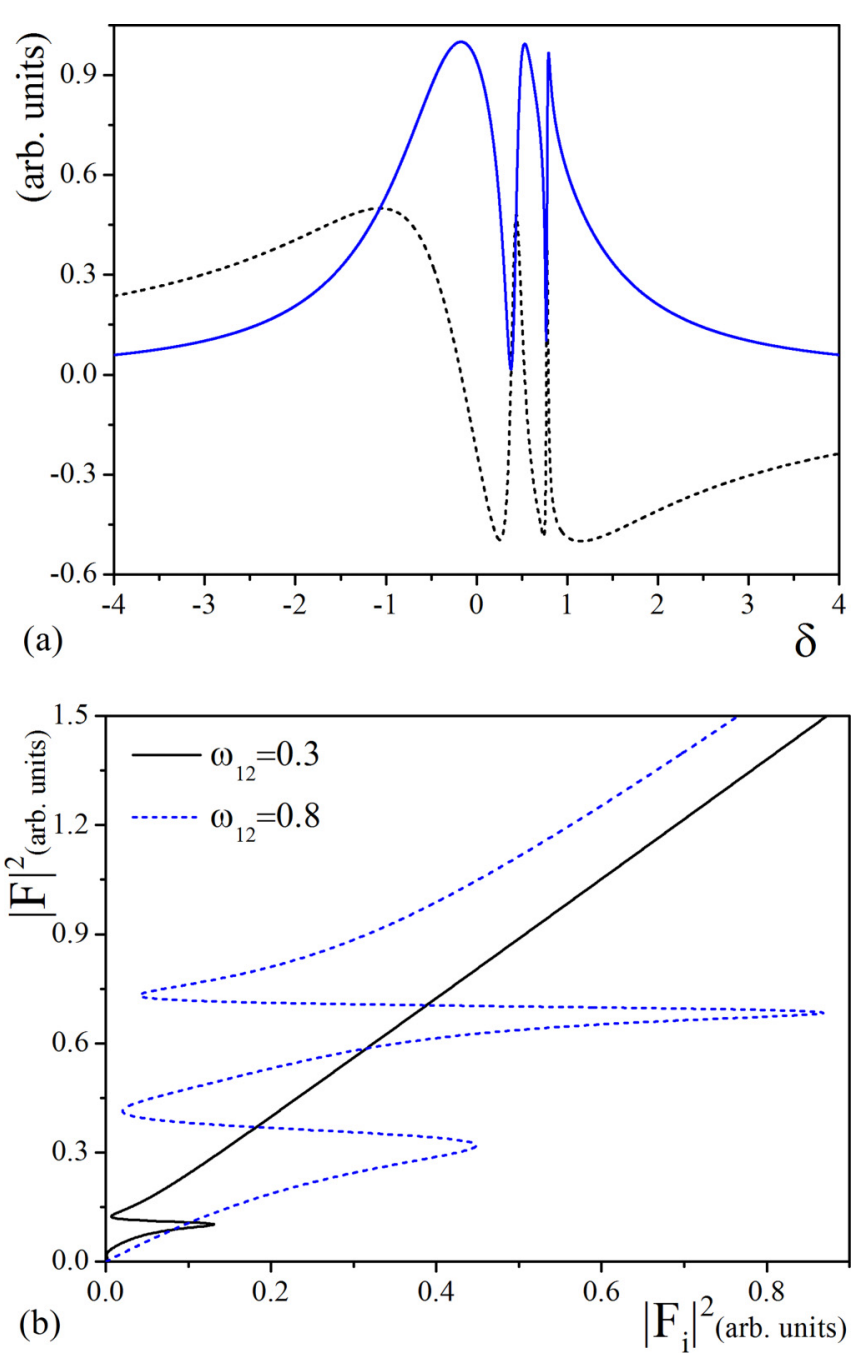

FIG. 2. (a) Imaginary (solid line) and real (dashed line) parts of the complex susceptibility $\chi$ for $\left|F_{i}\right|^{2}=0.5, T_{A}=0.1, T_{B}=0.01$, $\omega_{12}=0.67$, and $\omega_{23}=0.3$. (b) Bistability and multistability in the input-output intensities for $\Sigma=1, \delta=0.3$, and $\theta=-0.5$.

The linear stability of the homogeneous stationary solution is analyzed by studying the response of the system to small fluctuations around the steady state. We carry out a linear stability analysis by adding an ansatz of the form $F=$ $F_{s}+\delta F_{s} e^{\left(\lambda t-i\left(k_{x} x+k_{y} y\right)\right)}$, where $F_{s}, \delta F_{s}, \lambda$, and $k_{\perp}$ denote, respectively, the homogeneous stationary field, its deviation from the steady-state value, and the growth rates of the ansatz and transverse wave vectors. Once the ansatz is introduced into the homogeneous stationary equation, it can be solved along with its complex conjugate to obtain a characteristic equation (not presented here, for brevity) for the unstable spatial wave vectors. These wave vectors rule the periodicity of the incoming pattern in a mechanism typical of Turing pattern formation [20,21]. For this reason we label these instabilities Turing instabilities. In conservative systems like inviscid fluids or nonlinear Schrödinger equations in the presence of dispersion, similar instabilities have been called Benjamin-Feir or modulational instabilities. Our complex Eq. (1) is, however, dissipative and the presence of diffraction makes it mathematically equivalent to typical Turing diffusive
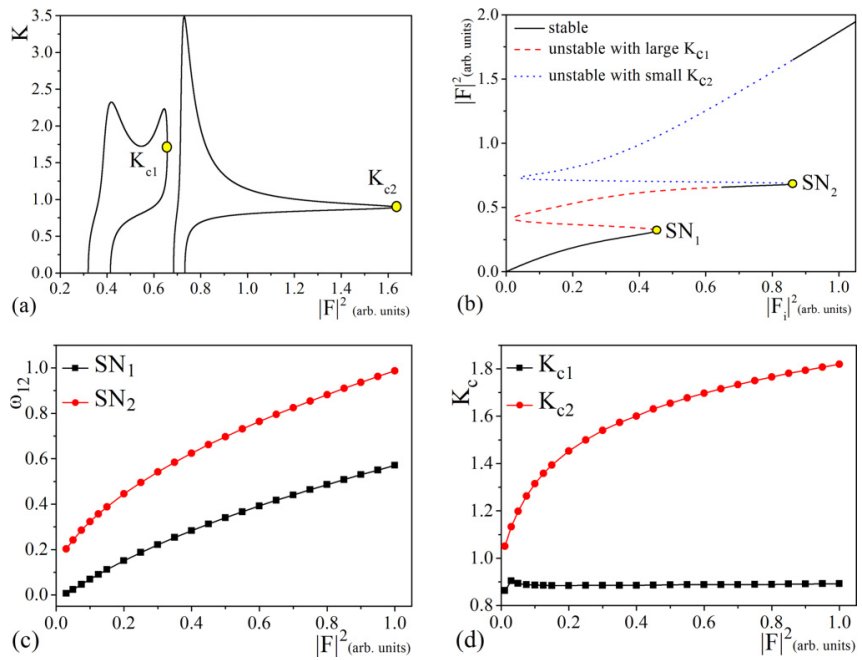

FIG. 3. (a) Turing instability domains having two different critical wave vectors, indicated by $K_{c 1}$ and $K_{c 2}$, and (b) their map on the multistability curve for $\omega_{12}=0.8$. (c) Variation of the control parameter threshold versus the stationary intensity for the onset of patterned solutions and (d) the corresponding critical wave vectors. Other parameters are the same as in Fig. 2.

systems as demonstrated in [21]. It is important to stress that the dynamic of the medium is considered much faster than that of the cavity field and that the explicit time dependence of matrix elements $\rho$ is not considered here.

For the parameters giving rise to multistable behavior, we expect separate Turing domains with different branches. In Fig. 3(a) we show the domains when $\omega_{12}$ is kept fixed at 0.8 and the pump intensity is swept instead. The map of the unstable domains on the multistability curve is shown in Fig. 3(b), where the simultaneous presence of Turing domains with different growth rates for the associated wave vectors is evident. Since we consider $\omega_{12}$ to be the control parameter rather than the pump intensity, the correspondence between $\omega_{12}$ and the stationary intensity $|F|^{2}$, which can effectively replace the pump intensity, is displayed in Figs. 3(c) and 3(d), where we show the saddle-node bifurcation points and critical wave vectors for the upper two branches. As shown, the threshold stationary intensity at which the homogeneous solutions bifurcate to spatially modulated ones increases when the control parameter $\omega_{12}$ is increased, meaning that more electron transfer from the first dot to the second (and hence to the third) delays the saturation. While this phenomenon occurs for both saddle-node points on the curve, the critical wave vector of the second instability domain is affected more by an increase in the population of second and third dots, whereas that of the first instability domain remains almost constant.

The instability ranges established via the linear stability of the homogeneous states are tested by numerical simulations in the next section.

\section{SPATIAL STRUCTURES}

The numerical code used for the dynamical simulation of Eq. (1) is based on a split-step method which solves the time derivative term by the Runge-Kutta algorithm and the diffraction by a fast Fourier transform algorithm. We used 

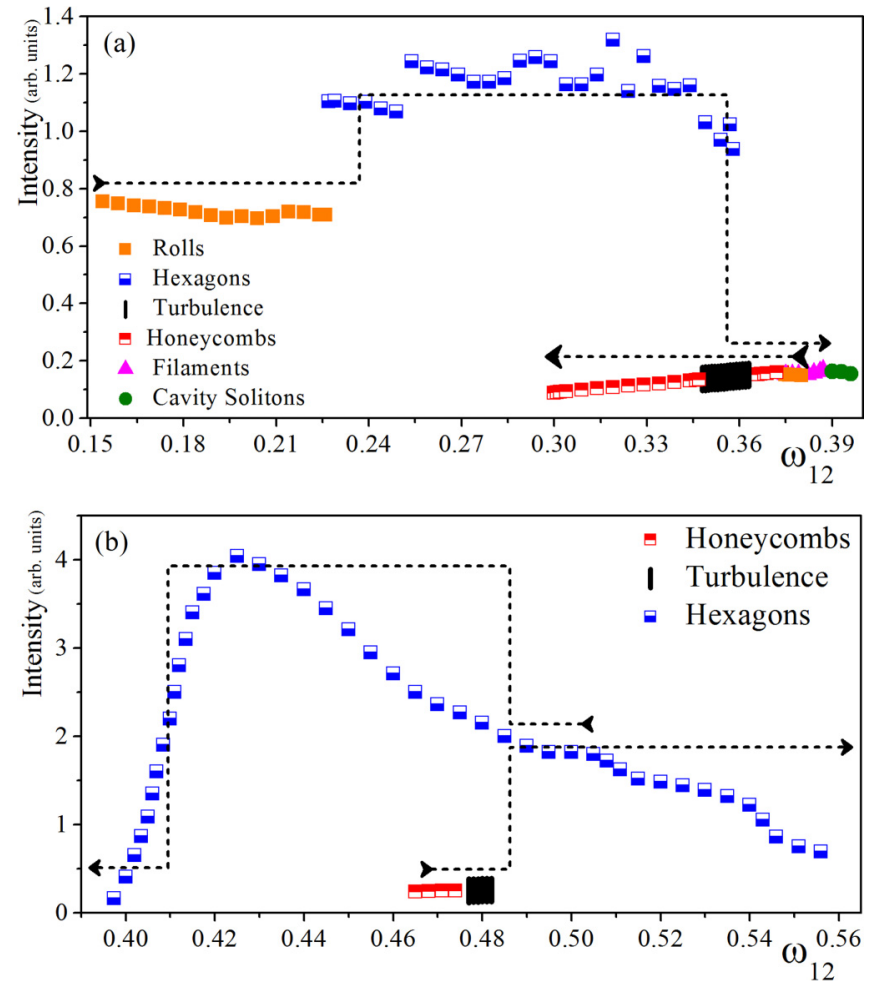

FIG. 4. Average intensity for a sequence of transverse spatial solutions for the instability domains of (a) the small and (b) the large wave vectors, respectively. The rightward and leftward arrows depict the direction of change in the control parameter $\omega_{12}$, implying scans I and II. Note that the trajectories of solutions in scans I and II depart from each other at $\omega_{12}=0.36$ and $\omega_{12}=0.485$ for the first and second instability domains, respectively.

$64 \times 64$ grids but checked the accuracy of the results with a larger numerical grid of $128 \times 128$ sites. We note that in the following we keep the stationary intensity $|F|^{2}$ fixed at 0.2 while changing the control parameter $\omega_{12}$, i.e., simulating the experimental situation of varying the gate voltage between the first and the second dots.

\section{A. Turing instability with a small critical wave vector}

With reference to the instability domains of different wave numbers in Fig. 3, two separate simulations were run for the middle and upper branches of the tristable curve corresponding to wave numbers $K_{c 1}$ and $K_{c 2}$. To run the simulations for the branch with a small critical wave vector $K_{c 2}$, we set the space step to be $10 \times 2 \pi / K_{c 2} \times 64$, where the critical wave number follows $K_{c 2}=\sqrt{0.285-\theta}$, corresponding to the instability domain of the uppermost branch. Starting from noise as the initial condition and by increasing the control parameter from a value well below the saddle-node point of the respective branch $\omega_{12}=0.13$ (scan I), a variety of transverse solutions develops as shown in Fig. 4(a). They begin with oscillating and scrolling rolls which lock at $\omega_{12}=0.21$ and change to oscillating hexagons at $\omega_{12}=0.23$. By further increasing the control parameter $\omega_{12}$, they lose their stability to a turbulent structure followed by a honeycomb solution. This scan finishes with wavy rolls and filaments which later break up into cavity solitons.
Also shown in Fig. 4(a) is a reverse scan of the same instability domain upon decreasing the control parameter, starting from $\omega_{12}=0.40$ and continuing beyond the point where homogeneous solutions have set in through scan I (see scan II). The transverse structures begin with regular rolls, coinciding with filaments of scan I, which change into honeycombs upon further decreasing the control parameter at $\omega_{12}=0.36$ and then to turbulent structures. We note that for this scan the sequence of the solutions departs from that of scan I from the middle of the turbulent structures' branch, and it settles in the homogeneous solution attractor after passing a series of honeycomb solutions. As we see later, the bistability of honeycomb and hexagonal solutions can explain both the oscillations in the peak intensity of the hexagonal patterns and the emergence of turbulent structures at the points where the trajectories of scans I and II separate.

\section{B. Turing instability with a large critical wave vector}

The same is done for the other instability domain using the large critical wave vector of $K_{c 1}=\sqrt{1.6-\theta}$. We observed an interval of honeycomb solutions changing into hexagons after a set of turbulent structures when we increased the control parameter starting from $\omega_{12}=0.462$ (scan I). In the reverse scan, starting from noise at $\omega_{12}=0.55$ and reducing the value of the control parameter (scan II), we only observed hexagonal structures forming from $\omega_{12}=0.50$ until the homogeneous state set in again. Figure 4(b) shows the sequence of the solutions in both scan I and scan II. The bistability between honeycomb and hexagonal solutions along with the turbulent range are once more the highlights of this instability domain and we note that scan II of this domain presents no turbulent structure. It should be remembered that the properties and characteristics of all the transverse patterns described here in the two cases of small and large critical wave numbers, except for the turbulent structures, have already been well documented in different contexts (see, for example, [20]).

\section{COMPETITION OF NONLINEARITIES AND OPTICAL TURBULENCE}

Hexagonal structures are common in pattern-forming systems and they come in two types: positive hexagons, with triangularly arranged peaks (intensity maxima) on a darker background; and honeycombs, which are dips (intensity minima) on a brighter background. In systems where a quadratic term governs the amplitude equations for the order parameter, the type of hexagons depends on the sign of such nonlinearity [22-27]. In particular, it has been shown that when spatial instabilities occur in a finite interval of a control parameter, the sign of the quadratic nonlinear term and thus the type of hexagonal structure can be different on opposite ends of the interval [26,27]. The formation of the two types of hexagonal patterns at the two ends of an instability interval has also been investigated in singly resonant parametric oscillators [10].

\section{A. Self-focusing and self-defocusing regimes}

For a Kerr medium, just one type of hexagons was observed in the presence of self-focusing nonlinearities [28-33]. It has been shown, however, that when moving from a self-focusing 


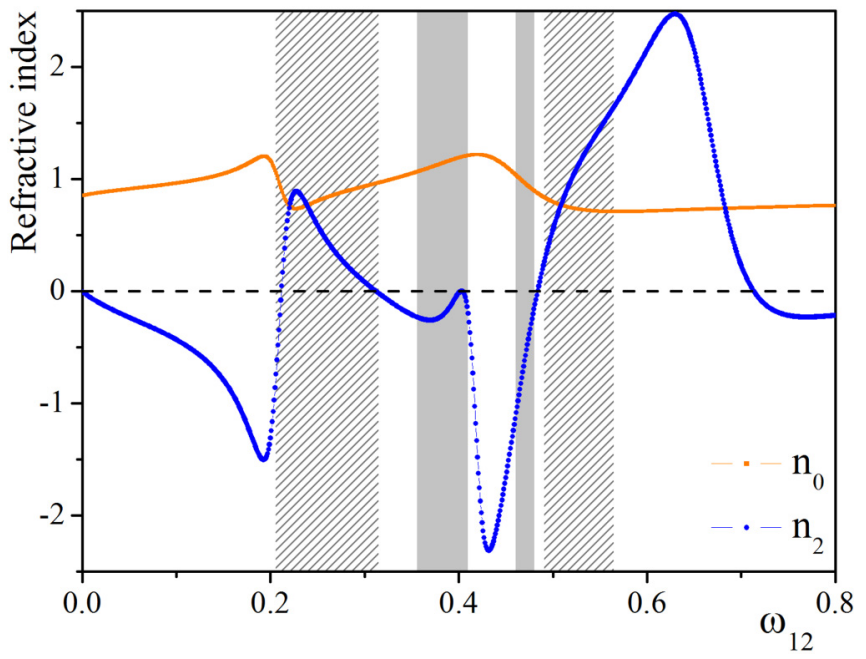

FIG. 5. Linear and nonlinear refractive indices versus increasing values of the control parameter $\omega_{12}$. Hatched areas show the $n_{2}>0$ region where hexagons form; gray shaded areas, honeycombs of $n_{2}<0$.

to a self-defocusing regime the sign of the quadratic nonlinearity in the amplitude equation changes [25] with a corresponding change in the type of hexagonal pattern generated. A straightforward transfer from a self-focusing to a self-defocusing regime has been demonstrated experimentally in electromagnetically induced transparency schemes based on $\Lambda$-type atomic systems $[34,35]$, where a clear change in sign of the Kerr-nonlinear coefficient $n_{2}$ was observed when passing from negative detuning values, $\delta$, to positive ones.

When scanning the control parameter $\omega_{12}$ we observe regimes dominated by either hexagons or honeycombs. More interestingly, we observe in both Fig. 4(a) and Fig. 4(b) regions where hexagons and honeycombs coexist. It is exactly in these regions where the sign of the Kerr nonlinearity changes and optical turbulence appears. The change in sign of the Kerr nonlinear coefficient $n_{2}$ is shown to occur as a result of the variations in the population of electrons occupying states $|2\rangle$ and $|3\rangle$, while the detuning value is fixed and the control parameter only changes the tunneling rate between states $|1\rangle \rightarrow|2\rangle$. Figure 5 shows that the nonlinear refractive index crosses 0 and changes sign in two different regions belonging to the two instability domains.

\section{B. Onset of turbulent structures}

When the system changes the sign of its nonlinearity $n_{2}$, the solutions pass through a region where turbulent structures form. In Figs. 6(a) and 6(b), the turbulent regions are shown in a plot of the wave number of the solutions versus the control parameter $\omega_{12}$. It is shown that at the two sides of the turbulence, there are bistabilities and competitions between two complementary hexagonal structures corresponding to opposite nonlinearities and having distinct wave numbers.

In Fig. 7, the transverse structures appearing before, within, and after the regions where the change in sign of $n_{2}$ takes place are shown. We observe that in both instability domains, there are turbulent solutions mediating the two
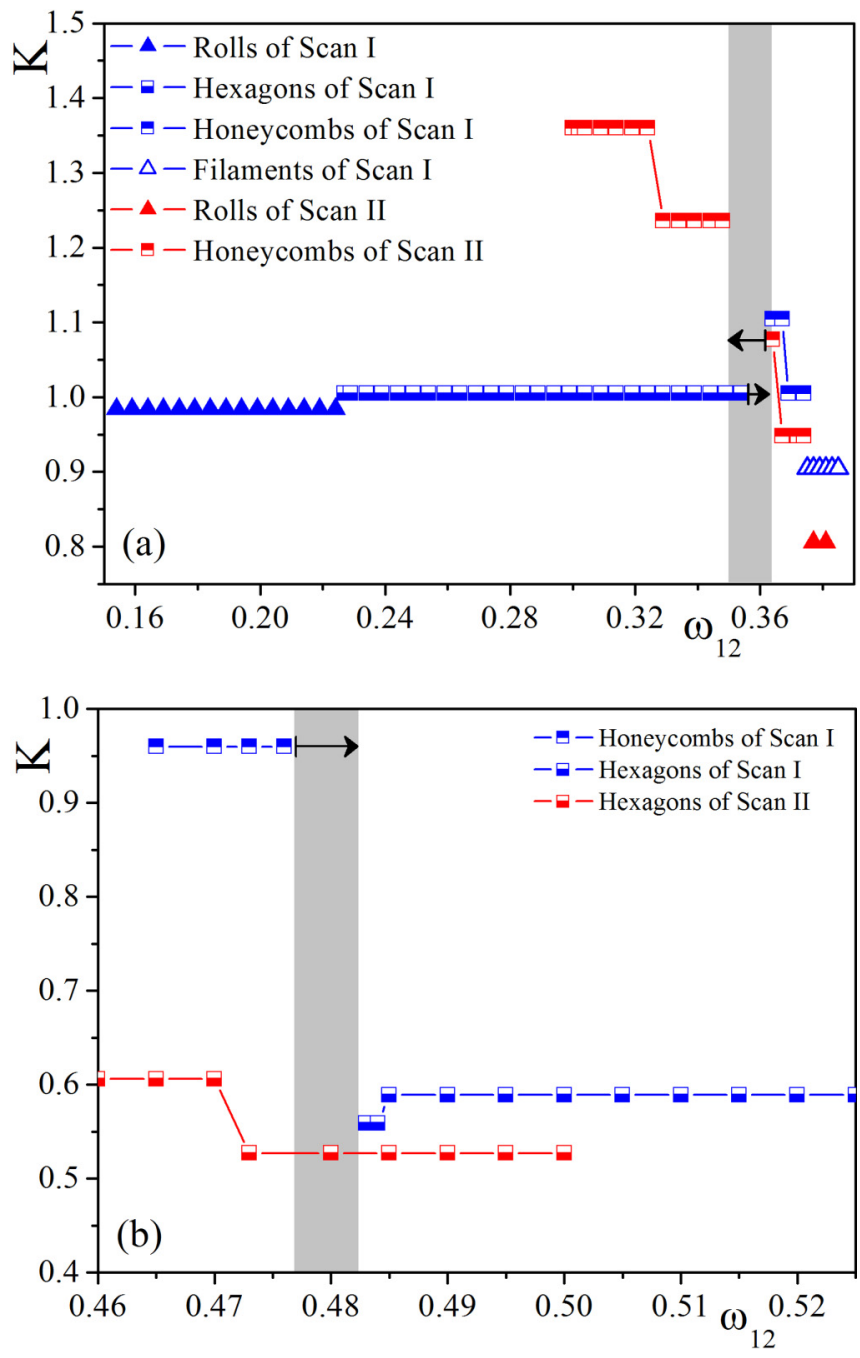

FIG. 6. Wave numbers associated with different solutions in different scans for the instability domains of (a) small and (b) large wave numbers. Gray areas depict the turbulent interval in either domain and the rightward (leftward) arrows show the direction of scan I (scan II). Note that for scan II in (b) no turbulent structure is excited.

complementary solutions at the moment of change in the sign of the nonlinearity.

Bistability between periodic Turing structures is less common than, for example, bistability or multistability of homogeneous solutions [15,36]. This is because, in general, stable Turing patterns are less common than homogeneous solutions and require their instability. In the case presented here the bistability is between the two complementary solutions characterized initially by a relatively large difference in their wave numbers. When changing the control parameter $\omega_{12}$ in both instability regions, however, the sizes of hexagons and honeycombs approach each other and competition for the same spatial regions ensues. This competition leads to the formation of turbulent structures characterized by irregular spatiotemporal dynamics. As shown in Fig. 8 for a simulation just above the threshold of the pattern bistability when the complementary pattern solution starts to grow, one single cell of the hexagonal pattern (i.e., an intensity peak) emerges 

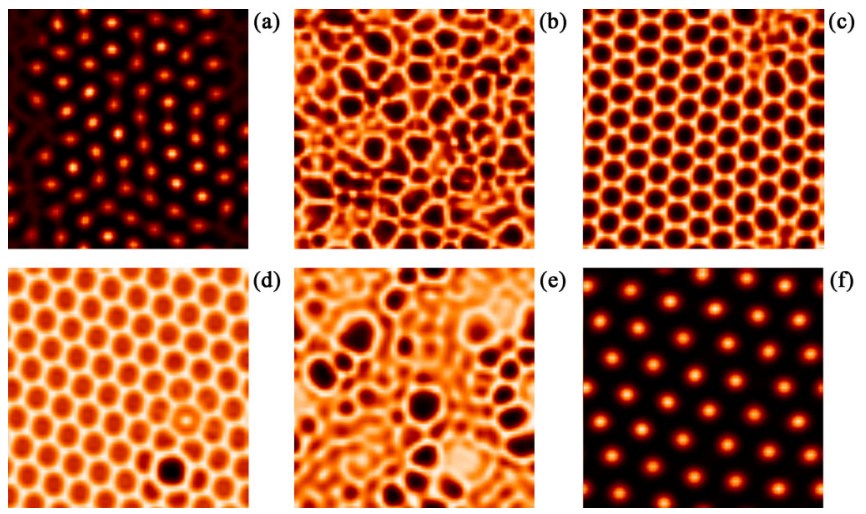

FIG. 7. Transverse structures (a), (d) slightly below, (b), (e) within, and (c), (f) slightly above the turbulent interval for the small (top row) and large (bottom row) wave-number instability domains.

in the intensity dip of the more stable honeycomb pattern. This competition between the two solutions corresponding to opposite nonlinearities results in the appearance and disappearance of localized cells of the less stable pattern within regions of the more stable structure. At the moment of its appearance (see Fig. 8) the emerging intensity peak pushes the boundaries of an already present honeycomb cell and creates a bigger hole. By increasing the control parameter and giving more strength to the emerging pattern, the number of peaks pushing away the walls of the background honeycomb pattern increases, and finally, a collapse of the domain wall separating the complementary structures takes place. It is here that one observes the onset of optical turbulence, which persists until the control parameter reaches a value where the emerging pattern becomes the dominant one and invades the entire transverse space.

The phenomenon here is rather different from what typically happens in fluid dynamics, where turbulent dynamics is always accompanied by energy transfer between larger and smaller scales of the motion through breakup of eddies as a result of a very high Reynolds number (the ratio of inertial forces to viscous forces within a fluid). This is known

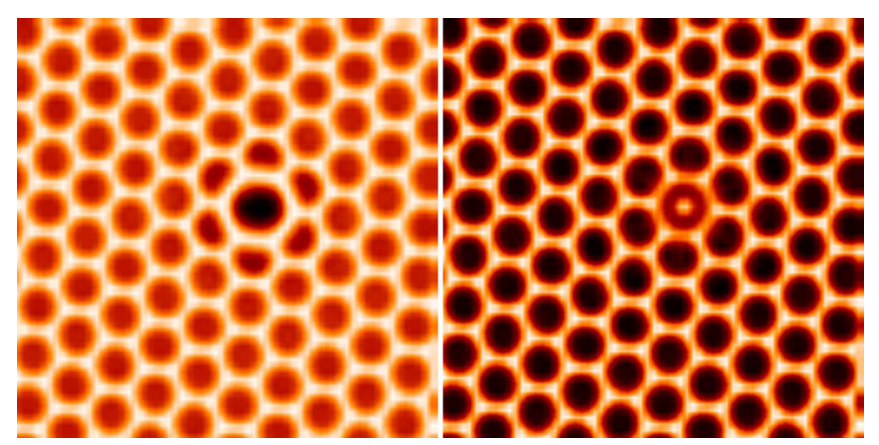

FIG. 8. Left: Widening of the intensity dip in the background pattern before the appearance of an intensity peak associated with the emerging pattern. Right: The same cell of the background pattern containing a peak of the new pattern, which shrinks after a short time when the emerging peak disappears. This breathing behavior continues until the control parameter is increased and more of these breathing cells engage in the competition. as Richardson cascade in fluid science and leads to a loss of spatial correlation and to an energy distribution function known as the Kolmogorov spectrum [37]. The essence of optical turbulence discussed here, however, corresponds to a loss of spatiotemproal correlation due to competition of spatial structures of similar spatial scales but with opposite nonlinearities. The energy flows between the two unstable structures and is dissipated via the cavity losses, which depend on the local value of the field amplitude.

\section{TRANSVERSE ROGUE WAVES}

It is interesting to investigate the intensity fluctuations when the hexagonal and honeycomb structures compete with each other. In the turbulent regime we observe from time to time the appearance of high-intensity peaks in the transverse plane. In transverse nonlinear optics, RWs have been described in optical parametric oscillators and laser systems [10-13] by extending methods developed for single transverse mode lasers with injected signals [4,5]. Transverse rogue wave events are pulses whose peak intensities are above the significant wave height, which is defined as the average pulse intensity, $\left\langle\langle I\rangle_{x, y}\right\rangle_{t}$, plus 4 times the standard deviation, $\sigma$, of the intensity fluctuations [38]. The significant wave height is between 5\% and $10 \%$ higher than the more classical definition of the mean wave height of the highest third of the waves and has been previously used in optics [5,39]. It has also been compared to a similar definition that uses the average pulse intensity plus 8 times the standard deviation $\sigma[4,10]$. We note that all the reported cases of transverse RWs in nonlinear optics are related to active systems with a threshold. Our triple-quantum-dot molecule device is instead a passive nonlinear optical cavity. Consequently, we do not require definitions of rogue waves that are more stringent than the significant wave height as adopted, for example, in [13], where extremely high events are due to laser Q-switchings, which are absent in our system. In Fig. 9 time traces of the maximum intensity, transverse average intensity, and standard deviation (from top to bottom) are shown in the left-hand panels for two dynamical evolutions in the optical turbulence regime $\left(\omega_{12}=0.359\right.$ and $\omega_{12}=0.363$, respectively). The corresponding probability density functions are displayed in the right-hand panels in Fig. 9.

To characterize the presence of RW events we have fitted the PDF data with Gaussian and Weibull distributions,

$$
\begin{gathered}
f(x)=a \exp \left[-\left(\frac{x-b}{c}\right)^{2}\right], \\
f(x)=c x^{b-1} \exp \left[-a x^{b}\right],
\end{gathered}
$$

respectively (see the solid red and dotted blue lines in the right-hand panels in Fig. 9). When the control parameter value is $\omega_{12}=0.359$ [Figs. 9(a) and 9(b)], we observe that the PDF has a Gaussian profile (best-fitting parameters $a=0.983$, $b=1.51, c=0.31)$, implying no RW events. As the control parameter is increased to $\omega_{12}=0.363$ [see Figs. 9(c) and 9(d)], the PDF clearly departs from a Gaussian profile (best-fitting parameters $a=0.6289, b=-1.14, c=0.88$ ) and displays a much longer tail containing pulses that satisfy the RW criterion (Weibull best-fitting parameters $a=31.42, b=-4.98$, $c=0.294$ ). 

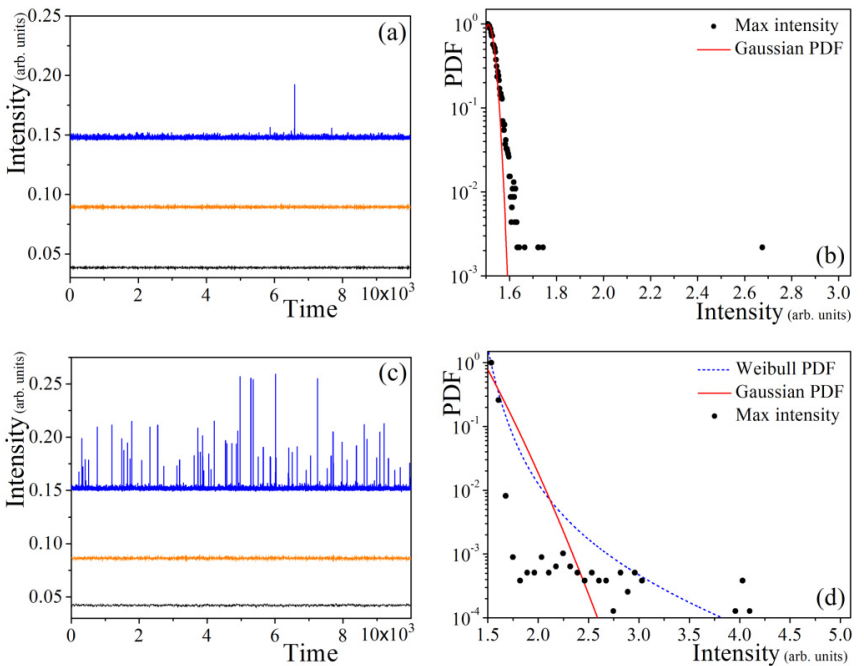

FIG. 9. Left: Maximum intensity (blue), transverse average intensity (orange) and standard deviation of the intensity (black) versus time, respectively, for $\omega_{12}=0.359$ and $\omega_{12}=0.363$, from top to bottom. Right: Corresponding PDF histograms for maximum intensity values. The solid red (dotted blue) line represents the best fit of the PDF data with a Gaussian (Weibull) distribution. Time is normalized to the photon lifetime in the cavity.

It is interesting to note that the extreme events that we observe during turbulence due to pattern competition tend to correspond to phase jumps of either $-\pi / 2$ or $\pi / 2$. In Fig. 10 we plot the intensities at the peaks versus their phase values for the turbulent regime without RWs (filled red circles), for the turbulent regime with RWs (blue circles) and for the honeycomb structure after the turbulent regime (green square). In all these cases, the spatial structures are phase bound, i.e., the phase values have a limited range. The persistence of phase-bound dynamics in the turbulent regimes is different from the rotating cases of RWs in defect-mediated turbulence presented in [11] for lasers with injected signals and

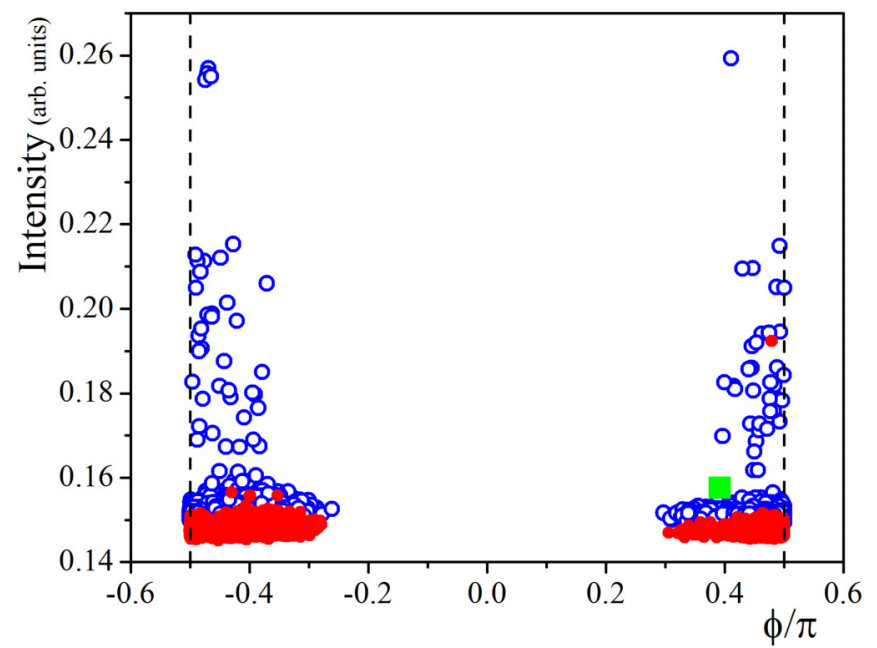

FIG. 10. Intensity versus phase values of the peaks. Filled red circles, turbulent structure with no RWs at $\omega_{12}=0.359$; blue circles, turbulent structure with RWs at $\omega_{12}=0.363$; and green square, stable honeycomb pattern at $\omega_{12}=0.370$.
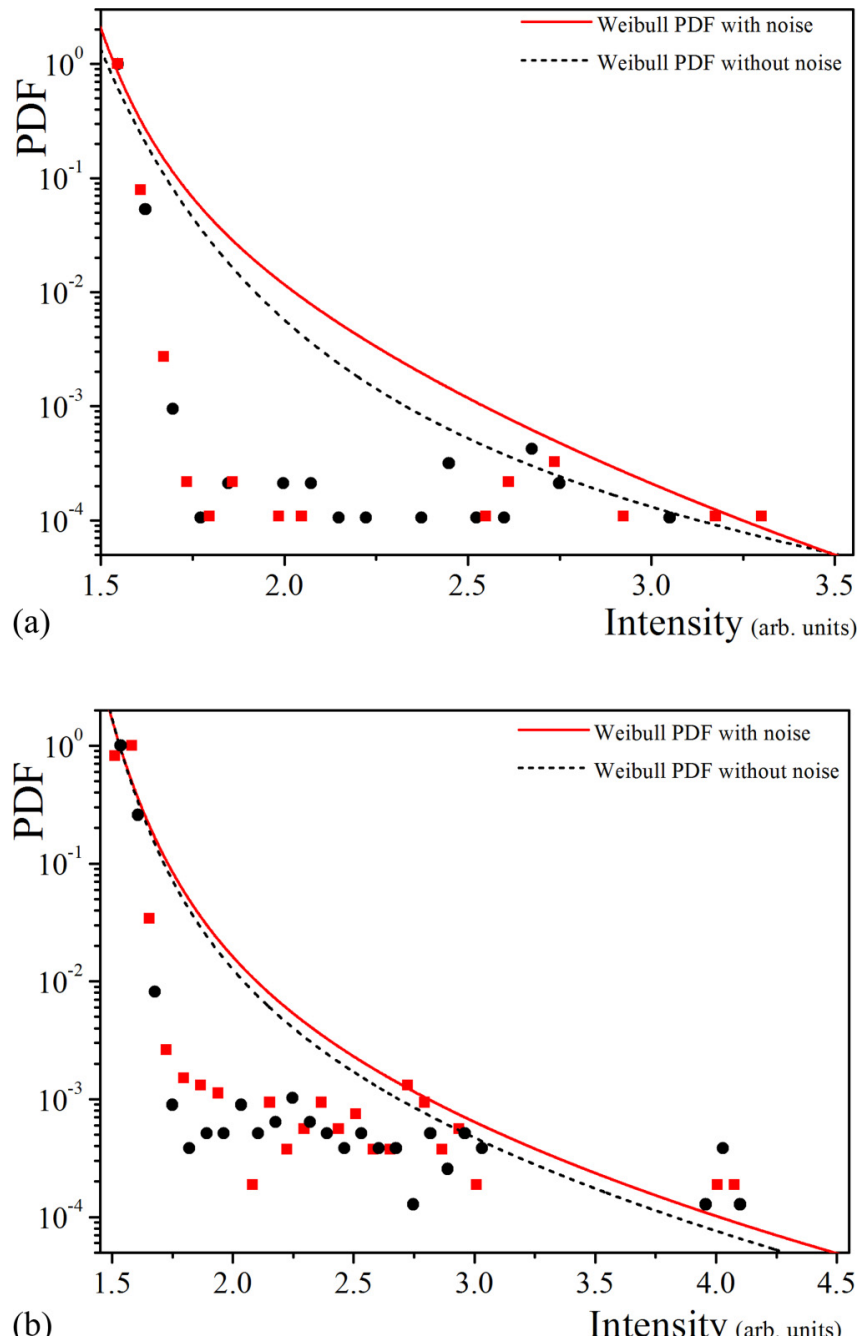

(b)

FIG. 11. The effect of noise on the probability density functions: (a) $\omega_{12}=0.361$ and (b) $\omega_{12}=0.363$. The curves represent the best Weibull fits with (solid red curve) and without (dotted black curve) noise.

optical parametric oscillators. When approaching the turbulent regime, the phase of the field tends to span a range between $-\pi / 2$ and $\pi / 2$. In Fig. 10 for $\omega_{12}=0.363$, we show that the maxima of the intensity corresponding to the RW peaks have either phase $\pi / 2$ or phase $-\pi / 2$. The distributions of phase and intensity values shrink to a small area when moving from the turbulent regimes to the stationary honeycombs (see the green square in Fig. 10).

As a final test of the robustness of the turbulent regimes, we added spontaneous emission noise to the dynamical equations to see if stochastic terms enhance or inhibit the occurrence of RWs. We have repeated the simulations for two control parameter values, $\omega_{12}=0.361$ and $\omega_{12}=0.363$, in the presence of spontaneous emission noise of amplitude $10^{-5}$ and in the turbulent regime [see Figs. 11(a) and 11(b), respectively]. The results show that the presence of noise does not alter the overall picture of rare events very much. However, we observe that for $\omega_{12}=0.361$, in the middle of the turbulent solutions interval, the addition of noise triggers more RWs, in contrast to $\omega_{12}=0.363$, where they are inhibited by the action 
of noise. This is somewhat expected since the latter lies just at the end of the turbulent range of solutions and the action of noise slightly drives the trajectory away towards the stable attractor belonging to a neighboring regular pattern.

\section{CONCLUSION}

The presence of spatiotemporal turbulence is a requirement for the observation of RWs in transverse nonlinear optics. Two-dimensional turbulence can have different origins: vorticity [40], underlying integrable dynamics [41], interacting vortices [11], and spatiotemporal chaos $[12,13]$. Here we have demonstrated that competition of transverse patterns through the simultaneous presence of focusing and defocusing nonlinearities first causes optical turbulence and then triggers RWs.

The turbulent states studied here owe their existence to the coherent manipulation of quantum states originating from conditions of tunneling-induced transparency in a cavity filled with molecules of triple quantum dots. This arrangement leads to a diversity of interesting phenomena including the simultaneous presence of Turing instability domains with different growth rates, multistability of steady states, pattern competition, and dynamical solutions. It is important to stress that the RWs described here are passive in nature since the system has no underlying gain or laser action.

We have further investigated the nature, properties, and robustness of the RWs in terms of probability distribution functions. Both periodic and turbulent structures are phasebound states, with RWs accumulating at phase values of $\pi / 2$ and $-\pi / 2$. We also demonstrated that the presence of realistic noise has no significant role in the number or distribution of optical RWs. We believe that the simplicity and efficiency offered by semiconductor nanostructures for coherent manipulations of quantum states along with the complexity of the phase space due to the cavity configuration make the triple-quantum-dot molecule system studied here an ideal candidate for the investigation of extreme events in solid-state devices.
[1] M. Onorato, S. Residori, U. Bortolozzo, A. Montina, and F. T. Arecchi, Phys. Rep. 528, 47 (2013).

[2] N. Akhmediev and E. Pelinovsky, Eur. Phys. J. Special Topics 185, 1 (2010).

[3] D. R. Solli, C. Ropers, P. Koonath, and B. Jalali, Nature 450, 1054 (2007).

[4] C. Bonatto, M. Feyereisen, S. Barland, M. Giudici, C. Masoller, J. R. Rios Leite, and J. R. Tredicce, Phys. Rev. Lett. 107, 053901 (2011).

[5] J. Zamora-Munt, B. Garbin, S. Barland, M. Giudici, J. R. Rios Leite, C. Masoller, and J. R. Tredicce, Phys. Rev. A 87, 035802 (2013).

[6] J. M. Dudley, F. Dias, M. Erkintalo, and G. Genty, Nat. Photon. 8, 755 (2014).

[7] C. Masoller, Y. Hong, S. Ayad, F. Gustave, S. Barland, A. J. Pons, S. Gómez, and A. Arenas, New J. Phys. 17, 023068 (2015).

[8] A. Hadjihosseini, M. Wächter, N. P. Hoffmann, and J. Peinke, New J. Phys. 18, 013017 (2016).

[9] N. Akhmediev, J. M. Soto-Crespo, and N. Devine, Phys. Rev. E 94, 022212 (2016).

[10] G.-L. Oppo, A. M. Yao, and D. Cuozzo, Phys. Rev. A 88, 043813 (2013).

[11] C. J. Gibson, A. M. Yao, and G.-L. Oppo, Phys. Rev. Lett 116, 043903 (2016).

[12] F. Selmi, S. Coulibaly, Z. Loghmari, I. Sagnes, G. Beaudoin, and M. G. Clerc, and S. Barbay, Phys. Rev. Lett. 116, 013901 (2016).

[13] C. Rimoldi, S. Barland, F. Prati, and G. Tissoni, Phys. Rev. A 95, 023841 (2017).

[14] G.-L. Oppo, J. Mod. Opt. 57, 1408 (2010).

[15] M. Eslami, R. Kheradmand, D. McArthur, and G.-L. Oppo, Phys. Rev. A 90, 023840 (2014).

[16] M. Scheibner, A. S. Bracker, D. Kim, and D. Gammon, Solid State Commun. 149, 1427 (2009).

[17] J. M. Villas-Boas, A. O. Govorov, and S. E. Ulloa, Phys. Rev. B 69, 125342 (2004).
[18] S.-C. Tian, R.-G. Wan, C.-Z. Tong, Y.-Q. Ning, L. Qin, and Y. Liu, J. Opt. Soc. Am. B 31, 1436 (2014).

[19] M. R. Mehmannavaz and H. Sattari, Laser Phys. Lett. 12, 025201 (2015).

[20] M. C. Cross and P. C. Hohenberg, Rev. Mod. Phys. 65, 851 (1993), and forward citations.

[21] G.-L. Oppo, J. Math. Chem. 45, 95 (2009).

[22] T. Ackemann, Yu. A. Logvin, A. Heuer, and W. Lange, Phys. Rev. Lett. 75, 3450 (1995).

[23] F. H. Busse, J. Fluid Mech. 30, 625 (1967).

[24] S. Ciliberto, P. Coullet, J. Lega, E. Pampaloni, and C. Perez-Garcia, Phys. Rev. Lett. 65, 2370 (1990).

[25] W. J. Firth, A. J. Scroggie, G. S. McDonald, and L. A. Lugiato, Phys. Rev. A 46, R3609(R) (1992).

[26] W. J. Firth and A. J. Scroggie, Europhys. Lett. 26, 521 (1994).

[27] M. Tlidi and P. Mandel, Chaos Solitons Fractals 4, 1475 (1994).

[28] G. D'Alessandro and W. J. Firth, Phys. Rev. A 46, 537 (1992).

[29] R. McDonald and H. J. Eichler, Opt. Commun. 89, 289 (1992).

[30] B. Thuring, R. Neubecker, and T. Tschudi, Opt. Commun. 102, 111 (1993).

[31] F. Papoff, G. D’Alessandro, G.-L. Oppo, and W. J. Firth, Phys. Rev. A 48, 634 (1993).

[32] E. Ciaramella, M. Tamburrini, and E. Santamato, Phys. Rev. A 50, R10 (1994).

[33] E. Pampaloni, S. Residori, and F. T. Arecchi, Europhys. Lett. 24, 647 (1993).

[34] H. Wang, D. Goorskey, and M. Xiao, Phys. Rev. Lett. 87, 073601 (2001).

[35] H. Wang, D. Goorskey, and M. Xiao, Opt. Lett. 27, 258 (2002).

[36] H. W. Xi, J. D. Gunton, and J. Vinals, Phys. Rev. E 47, R2987 (1993); D. Walgraef, Spatio-Temporal Pattern Formation 
(Springer-Verlag, Berlin, 1997); D. Michaelis, U. Peschel, and F. Lederer, Phys. Rev. A 56, R3366(R) (1997).

[37] U. Frisch, Turbulence: The Legacy of A. N. Kolmogorov (Cambridge University Press, Cambridge, UK, 1995).

[38] L. H. Holthuijsen, Waves in Oceanic and Coastal Waters (Cambridge University Press, Cambridge, UK, 2007), p. 70.
[39] C. Bonazzola, A. Hnilo, M. Kovalsky, and J. R. Tredicce, J. Opt. 15, 064004 (2013).

[40] A. Abrashkin and A. Soloviev, Phys. Rev. Lett. 110, 014501 (2013).

[41] P. Walczak, S. Randoux, and P. Suret, Phys. Rev. Lett. 114, 143903 (2015). 